\title{
A SAÚDE MENTAL DO ESTUDANTE DA GRADUAÇÃO: AÇÕES DE AMPARO PSICOSSOCIAL E PEDAGÓGICO
}

Tamara Silva de Paula - tamara.paula@engenharia.ufjf.br Universidade Federal de Juiz de Fora, Faculdade de Engenharia Campus sede UFJF - Rua José Lourenço Kelmer, s/n, São Pedro 36.036-900 - Juiz de Fora - MG

Yuri Mariano Carvalho - yuri.mariano@engenharia.ufjf.br Universidade Federal de Juiz de Fora, Faculdade de Engenharia Campus sede UFJF - Rua José Lourenço Kelmer, s/n, São Pedro 36.036-900 - Juiz de Fora - MG

Julia Righide Almeida-julia.righi@engenharia.ufjf.br Universidade Federal de Juiz de Fora, Faculdade de Engenharia Campus sede UFJF - Rua José Lourenço Kelmer, s/n, São Pedro 36.036-900 - Juiz de Fora - MG

LorenaGomes Abrantes - lorena.abrantes@engenharia.ufjf.br Universidade Federal do Rio de Janeiro, Coppe Av. Pedro Calmon, 550 - Cidade Universitária da Universidade Federal do Rio de Janeiro 21.941-901 - Rio de Janeiro - RJ

Resumo: O presente artigo busca abordar a necessidade de amparo psicossocial e pedagógico ao estudante do ensino superior, tratando, principalmente, dos graduandos de Engenharia. Para isso, através de discussão narrativa, este estudo apresenta dados referentes à saúde mental dos alunos e aos problemas ocasionados às Instituições de Ensino Superior decorrentes do mal-estar emocional, com enfoque na evasão. Em seguida, são apresentadas algumas ações já existentes, além da possibilidade das Metodologias de Aprendizagem Ativa como meio de amparo pedagógico.

Palavras-chave: Saúde Mental. Apoio Psicossocial. Apoio Pedagógico. Metodologias de Aprendizagem Ativa.

\section{INTRODUÇÃO}

A entrada na Universidade constitui um momento representativo na vida de muitos estudantes, marcando a conquista de um objetivo buscado, muitas vezes, ao longo de vários anos. Apesar disso, trata-se também de um período de importantes mudanças — como a saída da casa dos pais e a inserção em um novo meio - que podem gerar incertezas nos alunos (MARTINCOWSKI, 2013; SILVEIRA et al., 2011). Ao longo da graduação, o alto nível de exigência acadêmico e as metodologias de ensino arcaicas - além da preocupação com estágios, trabalhos de conclusão de curso e com a inserção no mercado - constituem novas fontes de estresse para os graduandos (CASTRO, 2017; MORETTI; HUBNER, 2017). 


\section{COBENGE 1110 e III Simpósio Internacional 2020}

Todo esse cenário ocasiona, além de dificuldades relacionadas ao bem-estar emocional dos estudantes, transtornos para as próprias Instituições de Ensino Superior (IES) - que podem resultar, por exemplo, em altos índices de evasão (ARAÚJO; BRESSAN, 2017; BUENO, 1993).

Dessa forma, na última década, é crescente a preocupação com a saúde mental dos alunos do ensino superior (ANDRADE et al., 2014; ARIÑO; BARDAGI, 2018; CASTRO, 2017; MORETTI; HUBNER, 2017; NOGUEIRA; SEQUEIRA, 2017; SILVEIRA et al., 2011), o que torna necessária a discussão sobre possibilidades de amparo aos graduandos, a fim de facilitar sua adaptação e permanência no meio acadêmico.

Nesse contexto, o presente trabalho busca apresentar meios de apoio aos discentes sob duas perspectivas: a psicossocial e a pedagógica. Para tal, é abordada, inicialmente, a problemática da saúde mental dos estudantes de graduação — especialmente a dos graduandos dos cursos de Engenharia - , contextualizando o período de entrada no ensino superior, as fontes de estresse, os fatores que os desmotivam durante o curso e os impactos negativos causado por esses fatores tanto em discentes quanto nas IES. Em seguida, o trabalho apresenta exemplos de apoio psicossociais desenvolvidos pelo Ministério da Educação (MEC) e pelas instituições de ensino e avalia, sob o ponto de vista pedagógico, o uso de Metodologias de Aprendizagem Ativa como forma de amparo à saúde mental dos estudantes, já que estas têm se mostrado eficazes na melhora do desempenho dos alunos e do interesse em sala de aula (DINIZ, 2009; FERREIRA et al., 2018; GUERRA; TEIXEIRA, 2016; TREVELIN; PEREIRA; OLIVEIRA NETO, 2013).

\section{METODOLOGIA}

Este artigo se caracteriza como uma discussão narrativa, tendo sido realizado através de análise qualitativa de dados secundários provenientes de artigos e de outras publicações técnico-científicas quanto à saúde mental de estudantes de graduação e possíveis formas de apoio psicológico e de melhoria pedagógica.

Optou-se por essa metodologia pelo fato de o tema em questão ser considerado atual e relevante face aos problemas verificados nas IES brasileiras, sendo necessário, portanto, buscar informações que ajudem na compreensão desse contexto.

Buscando melhor entendimento dessa problemática, são citados alguns exemplos de apoio psicossocial e pedagógico oferecidos em IES que têm se mostrado eficientes no auxílio aos estudantes.

\section{A SAÚDE MENTAL DO ESTUDANTE DE GRADUAÇÃO}

A entrada na Universidade constitui momento importante na vida do estudante, repleto de simbolismo social e realização familiar. Apesar disso, é também momento de mudanças, tomadas de decisões críticas e conflitos (MARTINCOWSKI, 2013).

Antes de escolher uma faculdade, o estudante precisa definir o caminho profissional que deseja seguir, algo nem sempre claro, e que pode trazer prejuízos à estabilidade e segurança na vida acadêmica. Ao ser aprovado, o aluno precisará desenvolver novas habilidades sociais, conhecendo um novo grupo de pessoas, novas metodologias de ensino e construir um perfil de estudante universitário. Este cenário de mudanças, que demanda adaptações frequentes por parte do aluno, pode se tornar fonte de estresse e impactar sua saúde (ARIÑO; BARDAGI, 2018; MARTINCOWSKI, 2013).

Durante a graduação, os fatores estressantes continuam e são vários (Figura 1). Uma pesquisa realizada com 82 alunos de diversos cursos de uma universidade particular de São Paulo, revelou que $89 \%$ consideram que a rotina universitária interfere negativamente em seus 
níveis de estresse, o que pode levar a um descontentamento com o curso escolhido, com os professores e com a própria instituição (MORETTI; HUBNER, 2017).

Figura 1 - Principais fontes de estresse durante a graduação

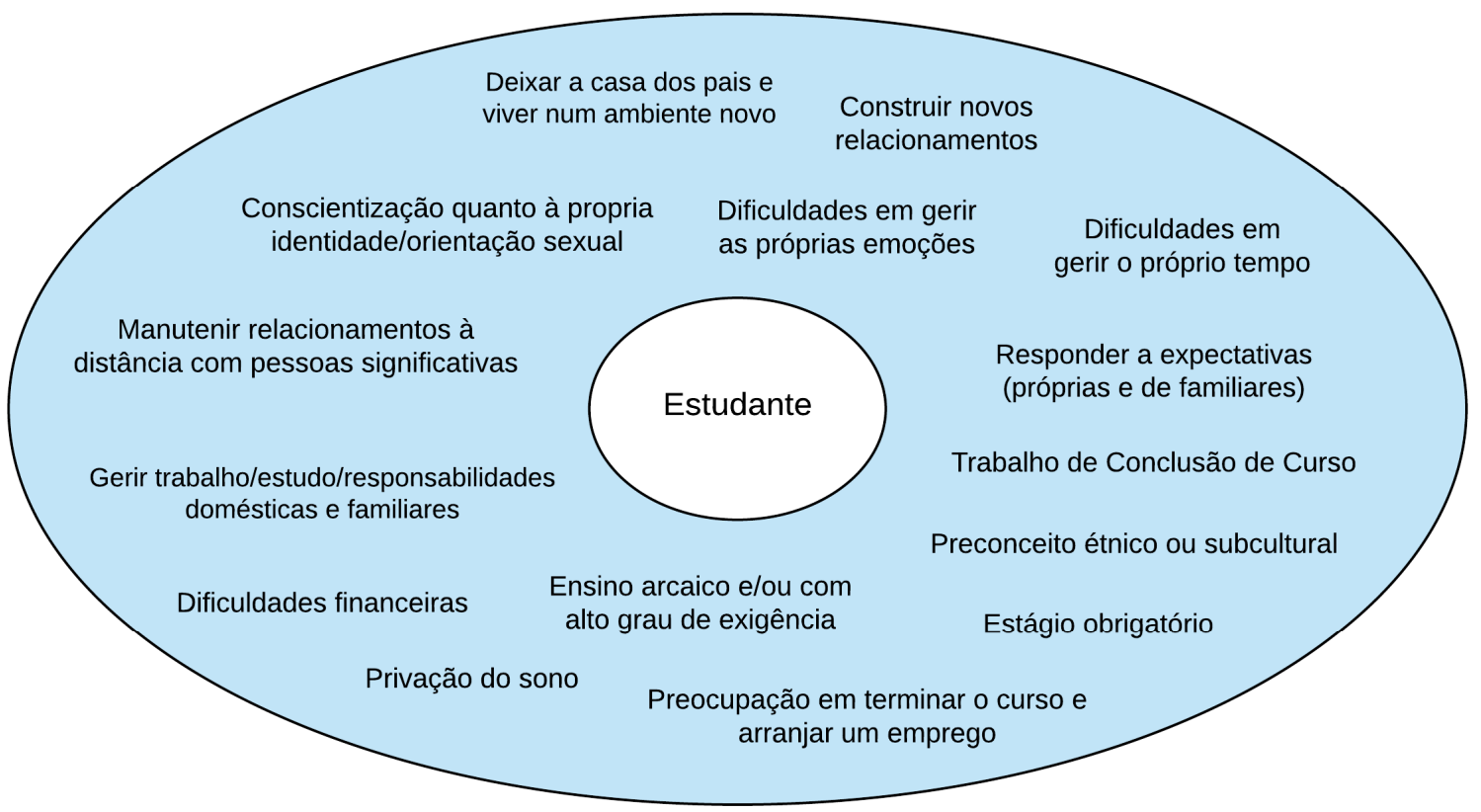

Fonte: adaptado de Castro (2017), Moretti e Hubner (2017) e Silveira et al. (2011).

Estudo semelhante foi realizado com 26 alunos dos períodos finais da graduação em Engenharia Civil da Universidade Federal de Itajubá, a partir da aplicação de questionários oriundos da área da Psicologia. Através do Inventário de Sintomas de Stress para Adultos de Lipp (ISSL), obteve-se que 62\% da amostra apresentava estresse; já segundo o Inventário de Depressão de Beck (BDI), obteve-se que 26,85\% da amostra estava incluída em faixas de depressão média e moderada; através do Inventário de Ansiedade de Beck (BAI), obteve-se que $31 \%$ da amostra possuía níveis de ansiedade; pelo questionário do Inventário de Ansiedade Traço-Estado (IDATE), obteve-se que mais de $80 \%$ da amostra estava incluída em faixas de ansiedade média e alta; e por fim, segundo o Maslach Burnout Inventory - Student Survey (MBI-SS), tem-se que 3,85\% da amostra manifestava o predomínio de burnout (CASTRO, 2017).

Além dos estudos já citados, os resultados obtidos na V Pesquisa Nacional de Perfil Socioeconômico e Cultural dos Graduandos das Instituições Federais de Ensino Superior Brasileiras (IFES), realizada pelo Fórum Nacional de Pró-Reitores de Assuntos Estudantis (FONAPRACE) - vinculado à Associação Nacional de Dirigentes das Instituições Federais de Ensino Superior (ANDIFES) (FONAPRACE/ANDIFES, 2019) - , com 424.128 discentes de cursos presenciais das 65 IFES existentes até fevereiro de 2018, mostraram que 83,5\% dos discentes experimentam algum tipo de dificuldade emocional que afeta sua vivência na graduação nos 12 meses anteriores à pesquisa. As maiores queixas dos estudantes referem-se à "[...] ansiedade, desânimo ou desmotivação, insônia ou alterações no sono, sensação de desamparo ou desespero, solidão e tristeza persistente." (FONAPRACE/ANDIFES, 2019). Com relação à pesquisa anterior (FONAPRACE/ANDIFES, 2016), realizada em 2014, houve uma piora na qualidade da saúde mental dos estudantes. A Tabela 1 mostra a estratificação dos dados apurados em 2018: 


\section{COBENCE 2020 da ABENGE}

"Os desafios para formar hoje o engenheiro do amanhã"

Tabela 1 - Percentual de alunos do ensino superior que apresentaram dificuldades emocionais nos doze meses anteriores à pesquisa

\begin{tabular}{c|c}
\hline Problemas ou sensações de: & Alunos que apresentam (em \%) \\
\hline Ansiedade & 63,6 \\
\hline Tristeza persistente & 22,9 \\
\hline Timidez excessiva & 16,2 \\
\hline Medo / pânico & 13,5 \\
\hline Insônia / Alterações no sono & 32,7 \\
\hline Desamparo / desespero & 28,2 \\
\hline Desatenção / desorientação / confusão mental & 22,1 \\
\hline Problemas alimentares & 12,3 \\
\hline Desânimo / desmotivação & 45,6 \\
\hline Solidão & 23,5 \\
\hline Ideia de morte & 10,8 \\
\hline Pensamento Suicida & 8,5 \\
\hline Dificuldades emocionais & 83,5 \\
\hline
\end{tabular}

Fonte: adaptado de V Pesquisa Nacional de Perfil Socioeconômico e Cultural dos Graduandos das IFES (2018).

Destaca-se que a porcentagem de alunos que possuem problemas relacionados à ansiedade representa, aproximadamente, $6 \mathrm{em}$ cada 10 estudantes. Os resultados indicaram ainda que, comparando com a IV Pesquisa, houve um aumento de 68,8\% na porcentagem de graduandos que têm pensamentos de morte e de $107,0 \%$ nos que possuem pensamento suicida (FONAPRACE/ANDIFES, 2016, 2019).

Os dados apurados na $\mathrm{V}$ Pesquisa mostraram também que a maioria dos discentes enfrenta, em média, 3 dificuldades simultaneamente e que "Entre os (as) estudantes que reportaram dificuldades emocionais $11,1 \%$ encontram-se em tratamento psicológico; $63,7 \%$ nunca procuraram atendimento psicológico; 7,5\% tomam medicação psiquiátrica." (FONAPRACE/ANDIFES, 2019).

Além das questões de cunho psicológico já apresentadas, as dificuldades de adaptação também podem levar à evasão de alunos no ensino superior (BUENO, 1993).

Com efeito, os resultados da V Pesquisa Nacional de Perfil Socioeconômico e Cultural dos Graduandos das IFES (FONAPRACE/ANDIFES, 2019) relacionados à evasão escolar, indicaram que 52,8\% dos alunos - sendo, deste total, 54,5\% graduandos em Engenharia já pensaram em abandonar seu curso. Quando indagados sobre os motivos (o questionário permitia assinalar uma ou mais alternativas),

[...] 32,8\% apontam as dificuldades financeiras, $29,7 \%$ o nível de exigência acadêmico, 23,6\% as dificuldades para conciliar os estudos e o trabalho, $21,2 \%$ os problemas de saúde, $19,5 \%$ as dificuldades do próprio campo profissional, $19,1 \%$ os relacionamentos no curso, $18,8 \%$ a incompatibilidade com o curso escolhido, $18,4 \%$ a insatisfação com a qualidade do curso, $15,9 \%$ os problemas familiares e 4,7\% assédio, bullying, perseguição, discriminação ou preconceito (FONAPRACE/ANDIFES, 2019, p.226).

Apesar do fator financeiro ser o mais crítico quando se apontam os motivos para evadir, o governo já busca, desde o período de ampliação do acesso ao ensino superior, destinar recursos para assistir os discentes, seja através do próprio Programa de Reestruturação e Expansão das Universidades Federais (REUNI), seja a partir do Programa Nacional de Assistência estudantil (PNAES) (FONAPRACE/ANDIFES, 2019). 
"Os desafios para formar hoje o engenheiro do amanhã"

Deste modo, é importante discutir a criação ou ampliação de políticas que deem suporte aos discentes nos demais "[...] aspectos acadêmicos, emocionais e institucionais que têm forte impacto na retenção e evasão.” (ARAÚJO; BRESSAN, 2017).

\section{APOIO PSICOSSOCIAL}

Na esfera jurídica, as atuais Diretrizes Curriculares Nacionais do Curso de Graduação em Engenharia (DCNs de Engenharia), estabelecem, em seu Art. $7^{\circ}$, que o "Projeto Pedagógico do Curso (PPC) deve prever os sistemas de acolhimento e nivelamento [dos estudantes], visando à diminuição da retenção e da evasão" (MEC/CNE/CES, 2019b). O acolhimento estudantil, por sua vez, caracteriza-se pela integração dos estudantes à instituição educacional, seja através de ações e atividades de cunho pedagógico ou não. Esse acolhimento pode ser mediado por professores, funcionários da própria instituição de ensino e, até mesmo, por outros estudantes (GIL et al., 2020). Nesse sentido, é válido citar algumas medidas que vêm sendo tomadas por IES brasileiras para melhorar o bem-estar emocional de seus graduandos.

Em 2011, durante o contexto de expansão e reestruturação, a Universidade Federal de Juiz de Fora (UFJF) criou a Pró-reitoria de Assistência Estudantil (PROAE). Composta por uma equipe multidisciplinar da qual fazem parte profissionais das áreas de serviço social, pedagogia, psicologia, além dos cargos administrativos, a PROAE tem como objetivo trabalhar na criação, implantação, administração e supervisão de políticas de apoio aos estudantes (DALESSI, 2018; UFJF, 2015; UFJF, 20--). O Plano de Desenvolvimento Institucional da UFJF (2015-2019), esclarece que:

Por apoio estudantil, compreende-se o enfrentamento de demandas
socioeconômicas dos discentes, para que a democratização do acesso ao
ensino superior seja acompanhada de efetivas possibilidades de permanência
dos estudantes; bem como o enfrentamento de demandas psicopedagógicas,
com o objetivo de que o nosso universo crescente de alunos possa se sentir
acolhido e reconhecido em sua diversidade e singularidades (UFJF, 2015,
p.107).

Além do atendimento pedagógico e psicológico, a PROAE desenvolve projetos como o grupo DeBoas, que busca instrumentalizar o estudante para que este lide melhor com a ansiedade, o Grupo Fora de Casa, que busca acolher os alunos vindos de cidades fora da localidade sede da UFJF, e o Grupo Roda Viva, que permite que os alunos que já estudam na instituição auxiliem no processo de adaptação dos calouros. Em 2017, a PROAE também foi umas das apoiadoras da $1^{\text {a }}$ Semana de Saúde Mental, organizada pelo Diretório Acadêmico da Faculdade de Engenharia da UFJF (DAEng/UFJF). Gratuito e aberto para o público em geral, o evento buscou propiciar aos estudantes um ambiente no qual foi possível compartilhar frustrações e falar sobre bem-estar emocional. O evento contou com momentos de música, prática de Yoga, além de rodas de conversa com psicólogos, discentes e profissionais da UFJF (UFJF, 2017; UFJF, 20--).

Outro exemplo de apoio aos estudantes é a Rede de Apoio ao Discente (RAD), criada na Universidade Federal de Alfenas (UNIFAL-MG), em 2016. A RAD conta com a participação de docentes, discentes e colaboradores externos de diversas áreas profissionais, entre elas enfermagem, pedagogia e psicologia, que buscam não só propiciar aos estudantes uma melhor vivência acadêmica, mas também diminuir os índices de evasão, contribuindo para o prosseguimento e finalização do curso (ARAÚJO; BRESSAN, 2017). 
"Os desafios para formar hoje o engenheiro do amanhã"

Para tanto, são desenvolvidas "[...] ações de promoção à saúde, atenção psicossocial e educacional aos discentes, em suas respectivas áreas ou de forma integrada, quando necessário.” (ARAÚJO; BRESSAN, 2017). A RAD disponibiliza as seguintes atividades:

[...] a acolhida ao calouro, serviço de acolhimento e Psicologia, rodas de Terapia Comunitária Integrativa, projeto Fala Garoto(a), oficinas de artesanato com material reciclável, rodas de conversa temáticas, ações de acessibilidade e inclusão, serviço de orientação educacional, atendimento por médico generalista, campanha Setembro Amarelo, parcerias interinstitucionais, mostra de talentos, criação de espaços de vivência, projeto UNIFAL-MG sem estresse, auxílio na divulgação de serviços e artigos produzidos pelos discentes, sensibilização do corpo docente e técnico sobre atitudes que geram opressão e traumas (ARAÚJO; BRESSAN, 2017, p.1).

\section{APOIO PEGAGÓGICO ATRAVÉS DE METODOLOGIAS DE APRENDIZAGEM ATIVA}

Sob o ponto de vista pedagógico, por sua vez, o uso de Metodologias de Aprendizagem Ativa em sala de aula tem se mostrado eficaz em aumentar os índices de aprovação e o interesse dos estudantes em aprender o conteúdo (DINIZ, 2009; FERREIRA et al., 2018; GUERRA; TEIXEIRA, 2016; TREVELIN; PEREIRA; OLIVEIRA NETO, 2013). Essas metodologias colocam o estudante como peça central no desenvolvimento das habilidades requeridas pela academia e pelo mercado de trabalho. Na engenharia, a expectativa é de que elas elevem a qualidade na forma de aprender dos alunos, fazendo com que o processo de aprendizagem vá além da retenção de tópicos e aumente o rendimento dos discentes, os índices de aprovação e, consequentemente, a permanência na Universidade (FERREIRA et al., 2018).

Uma pesquisa realizada com estudantes de Engenharia de Produção de uma instituição particular de ensino superior nas disciplinas de Cálculo II e Resistência dos Materiais, buscou comparar os níveis de aprovação e de satisfação dos alunos quando estes assistiam a aulas expositivas tradicionais e quando estes assistiam a aulas utilizando uma Metodologia de Aprendizagem Ativa. No caso do estudo, foi adotada a sala de aula invertida, metodologia que faz com que o aluno tenha contato prévio com o conteúdo da aula. Durante o período analisado, os professores indicavam alguns vídeos e textos no Ambiente Virtual de Aprendizagem e, ao iniciarem as aulas, aplicavam avaliações rápidas, que compunham a nota do aluno, e que abordavam o tema que seria discutido em sala (FERREIRA et al., 2018).

Os resultados da pesquisa indicaram não só a diminuição do índice de reprovação das disciplinas, como também o aumento na satisfação por parte dos estudantes, já que estes apontaram que a metodologia de aprendizagem ativa utilizada foi adequada para a exposição dos conteúdos, contribuiu para a sua formação e alcance de melhores resultados, além de incentivar a participação em sala de aula e comprometimento com as disciplinas (FERREIRA et al., 2018).

Estudo semelhante foi realizado com alunos do curso de Tecnologia em Análise e Desenvolvimento de Sistemas, da Faculdade de Tecnologia de Taquaritinga - CEETEPS, na disciplina Sistemas Operacionais I. A pesquisa buscou comparar os índices de aprovação e de reprovação entre três turmas onde o conteúdo foi ministrado através de aulas expositivas, com uma turma onde o modelo de sala de aula invertida foi adotado (TREVELIN; PEREIRA; OLIVEIRA NETO, 2013).

Assim como na pesquisa anterior, os alunos também tinham contato prévio com o conteúdo da aula, através da plataforma Moodle, onde o professor disponibilizava slides, links 
de sites, vídeos, atividades de fixação, além de demonstrar a aplicação do conteúdo no cotidiano. Em sala de aula, o professor apresentava os vídeos e exercícios propostos, de forma que a discussão teórica não ultrapassasse 20 minutos. Dessa maneira, as aulas se tornaram muito mais interativas, com resolução de dúvidas por parte não só do professor, mas também dos próprios alunos. Os resultados também mostraram aumento no índice de aprovação dos estudantes na disciplina (TREVELIN; PEREIRA; OLIVEIRA NETO, 2013).

Em outro estudo, a metodologia Problem Basead Learning (PBL), foi utilizada em quatro disciplinas do Curso de Engenharia de Materiais no Brasil. Os docentes dividiram as turmas em ilhas de aprendizagem, as quais foram apresentadas seis questões posteriormente respondidas por cada grupo de alunos. Além dos discentes terem mostrado melhora no desempenho acadêmico, eles também demonstraram maior motivação em sala de aula, provando a necessidade de se relacionar teoria e prática nos cursos, além da importância de aulas que fogem dos moldes tradicionais (TEIXEIRA; SILVA; BORGES, 2018).

A partir desses relatos sobre o uso de Metodologias de Aprendizagem Ativa, surge um questionamento válido que, potencialmente, ainda não é amplamente discutido no curso de graduação em Engenharia: como o uso da Aprendizagem Ativa pode contribuir para a melhoria da saúde mental dos estudantes?

\section{DISCUSSÃO}

Conforme exposto anteriormente, os estudantes do ensino superior estão sujeitos a uma série de fatores estressores durante o período universitário. Apesar de nos últimos anos ter havido alguns avanços no que diz respeito às políticas de apoio psicossocial nas IES - como a criação, por parte das próprias instituições de ensino, de ações de amparo ao discente e a expressa orientação nas DCNs de Engenharia sobre a necessidade de prover apoio psicossocial aos estudantes —, a maioria dos graduandos continua com dificuldades emocionais e não faz nenhum tipo de tratamento psicológico. É possível que os alunos não tenham conhecimento das ações de amparo desenvolvidas pela universidade, ou não se sintam à vontade para participar das atividades ofertadas pela instituição de ensino. Torna-se necessário, então, que se discuta a necessidade de criar, ampliar e disseminar medidas de apoio psicossocial nas instituições de ensino superior.

Considerando que muitos dos fatores que fragilizam a saúde mental de estudantes universitários estão associados ao ensino arcaico que vivenciam, o uso de metodologias de aprendizagem ativa desponta como uma das possíveis alternativas a ser adotada por professores universitários. Além dos benefícios para o aprendizado e para a real formação de competências pelos alunos, a adoção de metodologias de aprendizagem ativa aumenta a motivação dos estudantes, o que implica — além de melhor desempenho na disciplina menor evasão do curso de graduação.

Todavia, as estratégias e os métodos voltados para a aprendizagem ativa não devem substituir as ações desenvolvidas pelas universidades em prol do bem-estar emocional de seus estudantes, e sim serem adotadas como ferramentas psicopedagógicas que complementem as políticas de apoio à saúde mental dos alunos. Logo, os professores de IES devem ser capacitados para que, em suas práticas docentes, gerem ambientes agradáveis e atrativos para o aprendizado e que não afetem de forma negativa o psicológico do estudante.

\section{CONCLUSÃO}

Em síntese, o presente trabalho discutiu a problemática da saúde mental em estudantes de graduação - dando maior enfoque aos alunos do curso de Engenharia. Fica evidente ao longo do texto a necessidade de se criar e expandir o apoio psicossocial e pedagógico aos estudantes 
do ensino superior, visto que são inúmeros os fatores estressantes que prejudicam seu bemestar emocional.

Recomenda-se que sejam realizadas investigações e acompanhamentos junto aos alunos para avaliar os momentos pelos quais passam por maior vulnerabilidade ao longo da graduação. Dessa forma, a Instituição de Ensino Superior será capaz de melhor direcionar as ações de apoio e avaliar, nas instituições que já possuem programas de suporte, o porquê de, eventualmente, os graduandos se sentirem coagidos a procurar ajuda e as causas dessas ações não estarem contribuindo para a melhoria da saúde mental.

No que tange ao aspecto pedagógico, recomenda-se a realização de pesquisas quanto ao impacto dos diferentes tipos de Metodologias de Aprendizagem Ativa na saúde mental do estudante de Engenharia. Tais pesquisas deverão abordar, para além de variáveis quantitativas, como a nota dos discentes, a satisfação dos alunos ao vivenciar essas novas estratégias e métodos de aprendizagem e o quão preparado ele se sente para aplicar em sua realidade profissional os conteúdos e competências aprendidos em sala de aula, já que notas altas nem sempre representam aprendizado adequado.

Por fim, recomenda-se que as Instituições de Ensino Superior incentivem e/ou capacitem seu corpo docente para que sejam capazes tanto de aplicar estratégias e métodos de aprendizagem ativa em sala de aula quanto de direcionarem alunos que apresentem queixas e necessitem amparo psicossocial.

\section{REFERÊNCIAS}

ANDRADE, J. B. C. de et al. Contexto de formação e sofrimento psíquico de estudantes de medicina. Revista Brasileira de Educação Médica, v. 38, n. 2, 231-242, 2014.

ARAÚJO, C. L.; BRESSAN, Vânia R. Ações de promoção à saúde, atenção psicossocial e educacional como práticas de integração universitária. In: VII Conferencia Latinoamericana sobre el Abandono de la Educación Superior, 2017, Córdoba. Anais... Córdoba: Universidad Nacional de Córdoba; 2017.

ARIÑO, D. O.; BARDAGI, M. P. Relação entre Fatores Acadêmicos e a Saúde Mental de Estudantes Universitários. Revista Psicologia em Pesquisa, Juiz de Fora, v. 12, n. 3, p. 44$52,2018$.

BUENO, J. L. O. A evasão de alunos. Paidéia, Ribeirão Preto, n. 5, p. 9-16, 1993.

CASTRO, V. R. Reflexões sobre a saúde mental do estudante universitário: estudo empírico com estudantes de uma instituição pública de ensino superior. Revista Gestão em Foco, 9 ${ }^{\text {a }}$ edição, p. 380-401, 2017.

DALESSI, D. C. Política de assistência estudantil da UFJF e a comunidade acadêmica: limites e possibilidades dessa relação. 2018. 160 f. Dissertação (Mestrado em Gestão e Avaliação da Educação Pública) - Faculdade de Educação, Universidade Federal de Juiz de Fora, Juiz de Fora, 2018.

DINIZ, P. C. A. Utilização de rádio definido por software e metodologias ativas no ensino em engenharia elétrica. 2019. 110 f. Tese (Doutorado em Engenharia Elétrica) Universidade Federal de Uberlândia, Uberlândia, 2019. 
"Os desafios para formar hoje o engenheiro do amanhã"

FERREIRA, M. G. P. et al. Metodologias Ativas de Aprendizagem Aplicadas no Ensino da Engenharia. In: Congresso Internacional de Educação e Tecnologias - Encontro de Pesquisadores em Educação a Distância (CIET:EnPED). Anais... [S.I.], jun. 2018.

FONAPRACE/ANDIFES. IV Pesquisa Nacional de Perfil Socioeconômico e Cultural dos Graduandos da IFES. Uberlândia, jul. 2016. Disponível em: http://www.andifes.org.br/wpcontent/uploads/2017/11/Pesquisa-de-Perfil-dos-Graduanso-das-IFES_2014.pdf. Acesso em: 13 jul. 2020.

\section{Pesquisa Nacional de Perfil Socioeconômico e Cultural dos Graduandos da} IFES. Uberlândia, mai. 2019. Disponível em: http://www.andifes.org.br/wpcontent/uploads/2019/05/V-Pesquisa-do-Perfil-Socioecon\%C3\%B4mico-dos-Estudantes-deGradua\%C3\%A7\%C3\%A3o-das-Universidades-Federais-1.pdf. Acesso em: 13 jul. 2020.

GIL, H. A. C. et al. Acolhimento do aluno ingressante nos cursos de Engenharia. In: OLIVEIRA, V. F. (org). A Engenharia e as novas DCNs: oportunidades para formar mais e melhores engenheiros. $1^{\text {a }}$ ed. Rio de Janeiro: LTC, 2019. p. 182-197.

GUERRA, C. J. O.; TEIXEIRA, A. J. C. Os impactos da adoção de metodologias ativas no desempenho dos discentes do curso de ciências contábeis de instituição de ensino superior mineira. Revista de Educação e Pesquisa em Contabilidade (REPeC), v. 10, n. 4, art. 2, p. 380- 397, 2016.

MARTINCOWSKI, T. M. A inserção do aluno iniciante de graduação no universo autoral: a leitura interpretativa e a formação de arquivos. Cadernos da Pedagogia, São Carlos, Ano 6 v. 6 , n. 12, p.129-140, 2013.

MEC/CNE/CES. Resolução $n^{\circ}$ 2, de 24 de abril de 2019. Institui as Diretrizes Curriculares Nacionais do Curso de Graduação em Engenharia. Diário Oficial da União, 80 ed., seção 1, p. 43.

MORETTI, F. A.; HÜBNER, M. M. C. O estresse e a máquina de moer alunos do ensino superior: vamos repensar nossa política educacional? Revista Psicopedagogia, v. 34, n. 105, p. $258-267,2017$.

NOGUEIRA, M. J.; SEQUEIRA, C. A saúde mental em estudantes do ensino superior: relação com o género, nível socioeconômico e os comportamentos de saúde. Revista Portuguesa de Enfermagem de Saúde Mental, Porto, n. spe5, p. 51-56, 2017.

SILVEIRA, C. et al. Saúde mental em estudantes do ensino superior: experiência da consulta de Psiquiatria do Centro Hospitalar São João. Acta Médica Portuguesa, 24(S2), p. 247-256, 2011.

TEIXEIRA, R. L. P.; SILVA P. C. D.; BRITO. M. L. de A. Aplicabilidade de metodologias ativas de aprendizagem baseada em problemas em cursos de graduação em engenharia. Revista Humanidades e Inovação, v. 6, n. 8, p. 138-147, 2019. 
TREVElin, A. T. C.; PEREIRA, M. A. A.; OliveIRA NETO, J. D. de. A utilização da "sala de aula invertida" em cursos superiores de tecnologia: comparação entre o modelo tradicional e o modelo invertido "flipped classroom" adaptado aos estilos de aprendizagem. Revista de Estilos de Aprendizagem, Madrid, v. 6, n. 12, out. 2013.

UFJF. Plano de Desenvolvimento Institucional - 2015-2019. Juiz de Fora, dez. 2015. Disponível em: https://www2.ufjf.br/ufjf/files/2016/01/pdi_20162020_ufjftexto_aprovado.pdf. Acesso em: 22 jul. 2020.

Diretório Acadêmico da Engenharia organiza Semana da Saúde Mental. 15 de setembro de 2017. Disponível em: http://www.ufjf.br/noticias/2017/09/15/diretorioacademico-da-engenharia-organiza-semana-da-saude-mental/. Acesso em: 22 jul. 2020.

- Pró-Reitoria de Assistência Estudantil. Disponível em: https://www2.ufjf.br/proae/. Acesso em: 23 jul. 2020. 20--.

\title{
COLLEGE STUDENT MENTAL HEALTH: PSYCHOSOCIAL AND PEDAGOGICAL SUPPORT ACTIONS
}

\begin{abstract}
This paper seeks to address the need of psychosocial and pedagogical support for college students, dealing mainly with Engineering undergraduates. For this, through the narrative discussion, this study presents data related to the students' mental health and the problems caused to the Higher Education Institutions due to the emotional malaise, with a focus on evasion. Then, some existing actions are included, besides the possibility of Active Learning Methodologies as a means of pedagogical support.
\end{abstract}

Keywords: Mental health. Psychosocial Support. Pedagogical Support. Active Learning Methodologies. 